\title{
Osons la différence
}

\author{
par Tania Mysak
}

$\mathrm{Q}$ uand ce numéro sera publié, le conseil d'administration de la Société canadienne des pharmaciens d'hôpitaux (SCPH), les présidents des sections et les présidents des conseils d'administration affiliés auront terminé une séance de planification stratégique. Si vous avez déjà participé à une planification stratégique, peut-être éprouvez-vous des sentiments partagés à l'égard de cette expérience. Vous aimez l'énergie qui consiste à viser les étoiles en créant des occasions faisant progresser votre organisation. Vous détestez forger des énoncés et des objectifs idéalistes qui obligent à des actions concrètes et mesurables privées de l'étincelle de l'inspiration. Un plan stratégique bien conçu doit imprégner toute l'organisation; tout le monde doit ramer dans la même direction pour atteindre un objectif commun. Quand la haute direction articule un travail progressif en vue d'atteindre des objectifs, il est trop fréquent de voir les membres de première ligne qui ne savent pas articuler le plan, ce qui démontre une rupture du degré d'engagement et du sens de l'objectif commun. Étant donné ces limites actuelles, la SCPH ne peut pas maintenir le statu quo en matière de planification stratégique.

Conscients des changements importants à apporter au sein de la SCPH pour soutenir l'organisation, nous avons décidé d'aborder notre séance de planification stratégique un peu différemment. Nous définissons généralement une, vision et un énoncé de mission, puis nous réfléchissons à des objectifs et nous développons des stratégies ou des tactiques pour les atteindre. Mais que faire si on expose déjà le travail véritablement crucial qui s'annonce alors que l'ancienne vision et l'ancienne mission résonnent encore largement dans l'esprit des membres ?

En tant que Société, nos responsabilités fondamentales visent à articuler une mission et une vision qui trouvent un écho chez les membres et qui permettent d'exercer une gouvernance efficace tout en assurant une infrastructure qui soutienne notre travail. Dans notre dernier sondage mené auprès des membres, $84 \%$ d'entre eux convenaient (ou étaient fortement d'accord) que nos énoncés de mission et de vision actuels devaient évoluer. Concernant la gouvernance et l'infrastructure, ce travail a été approuvé dans le plan Stratégie en vue de la pérennisation (Strategy Towards Sustainability; https://www.cshp.ca/strategy- towards-sustainability) et il incombe au chef de la direction et au personnel.

Notre activité principale se reflète dans les rôles pour lesquels nous sommes reconnus, mais aussi dans ceux que les membres jugent appropriés de la part de la SCPH. Répétons-le, notre conseil d'administration a dégagé un consensus validé par le sondage mené auprès des membres, voulant que la formation, la pratique et les normes professionnelles, la défense des intérêts, le Journal canadien de la pharmacie hospitalière et les services aux membres constituent l'essence même de la SCPH. Avoir de solides fondations et focaliser notre travail sur ces activités centrales nous permettent d'offrir de la valeur aux membres et de répondre aux besoins qu'ils ont exprimés.

Enfin, ayant jeté des bases solides tout en restant concentrés sur les éléments vraiment importants pour la $\mathrm{SCPH}$, nous pouvons nous pencher sur les stratégies qui soutiendront l'organisation et permettront de la développer dans les années à venir. Ce sont ces «grandes idées» qui permettront à la SCPH de passer à l'étape suivante et de poursuivre sa progression, selon les discussions qui se sont déroulées dans le cadre du conseil d'administration au moment de l'élaboration du plan Stratégie en vue de la pérennisation (Strategy Towards Sustainability), lequel se concentre sur l'adhésion des techniciens en pharmacie, la spécialisation et le changement de nom de la Société. Nous avons testé ces idées dans le sondage mené auprès des membres. Notre séance de planification stratégique a été l'occasion d'approfondir ces idées et de réfléchir à la manière de faire évoluer la SCPH vers un objectif d'adhésion et de durabilité financière.

Nous vous ferons part des résultats de notre séance au cours des mois à venir et nous nous réjouissons de recevoir vos commentaires pour savoir si notre approche « différente» de cette année est la bonne!

[Traduction par l'éditeur]

Tania Mysak, BSP, Pharm. D., est présidente et agente de liaison pour la vision de la Société canadienne des pharmaciens d'hôpitaux. 